\title{
Improvement in Visual Search with Practice: Mapping Learning-Related Changes in Neurocognitive Stages of Processing
}

\author{
Kait Clark, ${ }^{1,3}$ L. Gregory Appelbaum, ${ }^{1,4}$ Berry van den Berg, ${ }^{1,5}$ Stephen R. Mitroff, ${ }^{1,2}$ and Marty G. Woldorff ${ }^{1,2,4}$ \\ ${ }^{1}$ Center for Cognitive Neuroscience and ${ }^{2}$ Department of Psychology and Neuroscience, Duke University, Durham, North Carolina 27708, ${ }^{3}$ School of \\ Psychology, Cardiff University, Cardiff CF10 3AT, United Kingdom, ${ }^{4}$ Department of Psychiatry, Duke University Medical Center, Durham, North Carolina \\ 27708, and ${ }^{5} \mathrm{BCN}$ Neuroimaging Center, University of Groningen, $9700 \mathrm{AB}$ Groningen, The Netherlands
}

Practice can improve performance on visual search tasks; the neural mechanisms underlying such improvements, however, are not clear. Response time typically shortens with practice, but which components of the stimulus-response processing chain facilitate this behavioral change? Improved search performance could result from enhancements in various cognitive processing stages, including (1) sensory processing, (2) attentional allocation, (3) target discrimination, (4) motor-response preparation, and/or (5) response execution. We measured event-related potentials (ERPs) as human participants completed a five-day visual-search protocol in which they reported the orientation of a color popout target within an array of ellipses. We assessed changes in behavioral performance and in ERP components associated with various stages of processing. After practice, response time decreased in all participants (while accuracy remained consistent), and electrophysiological measures revealed modulation of several ERP components. First, amplitudes of the early sensoryevoked $\mathrm{N} 1$ component at $150 \mathrm{~ms}$ increased bilaterally, indicating enhanced visual sensory processing of the array. Second, the negativepolarity posterior-contralateral component $(\mathrm{N} 2 \mathrm{pc}, 170-250 \mathrm{~ms})$ was earlier and larger, demonstrating enhanced attentional orienting. Third, the amplitude of the sustained posterior contralateral negativity component (SPCN, $300-400 \mathrm{~ms}$ ) decreased, indicating facilitated target discrimination. Finally, faster motor-response preparation and execution were observed after practice, as indicated by latency changes in both the stimulus-locked and response-locked lateralized readiness potentials (LRPs). These electrophysiological results delineate the functional plasticity in key mechanisms underlying visual search with high temporal resolution and illustrate how practice influences various cognitive and neural processing stages leading to enhanced behavioral performance.

Key words: attention; EEG; learning; LRP; N2pc; visual search

\section{Introduction}

Visual search, the process of detecting target items among distractors, is a vital cognitive ability central to many everyday human activities, as well as to societally critical job tasks such as detecting abnormalities in radiological images and screening airport luggage for contraband (for review, see Clark et al., 2012; Wetter, 2013). Successful search requires the execution of a cascade of fundamental cognitive processes, including sensory analysis of the scene, orienting of visual attention, working memory, target discrimination, and decision/response processes (for a re-

Received March 21, 2014; revised Dec. 11, 2014; accepted Jan. 22, 2015.

Author contributions: K.C., L.G.A., S.R.M., and M.G.W. designed research; K.C., L.G.A., and M.G.W. performed research; K.C., L.G.A., and B.v.d.B. analyzed data; K.C., L.G.A., S.R.M., and M.G.W. wrote the paper.

This work was supported by U.S. NIH Grant R01-MH060415 to M.G.W, with some support from U.K. ESRC/ORA+ Grant ES/M00001X/1.

The authors declare no competing financial interests.

This article is freely available online through the J Neurosci Author Open Choice option.

Correspondence should be addressed to Marty G. Woldorff, Center for Cognitive Neuroscience, Duke University, Box 90999, Durham, NC 27708. E-mail: woldorf@ @duke.edu.

DOI:10.1523/JNEUROSCI.1152-14.2015

Copyright $\odot 2015$ Clark et al.

This is an Open Access article distributed under the terms of the Creative Commons Attribution License Creative Commons Attribution 4.0 International, which permits unrestricted use, distribution and reproduction in any medium provided that the original work is properly attributed. view, see Eckstein, 2011; Nakayama and Martini, 2011). These cognitive faculties are supported by various underlying neural mechanisms ranging from low-level feature analyses to higher-level, goal-driven decision processes (Treisman and Gelade, 1980; Duncan and Humphreys, 1989; Corbetta and Shulman, 2002).

Prior research has established that visual search can be improved through experience or practice (Sireteanu and Rettenbach, 1995; Sigman and Gilbert, 2000). Given the numerous neurocognitive stages involved in detecting, assessing, and responding to search stimuli, questions remain as to which processes are enhanced and in what relative combination. Several recent visual search studies have reported amplitude changes in certain scalp-recorded event-related potential (ERP) components after practice on complex conjunction search tasks (Hamame et al., 2011; An et al., 2012); however, the learning-related changes underlying training-induced behavioral improvement in the rapid, tightly timed, parallel processing involved in feature-popout search tasks are unclear.

Here, we investigated changes across the entire stimulusresponse processing cascade that underlies popout visual search learning by leveraging the high temporal resolution of ERPs elicited while searching visual arrays containing rapidly processed feature-popout targets. Participants completed a five-day behavioral practice protocol (with electrophysiological activity re- 


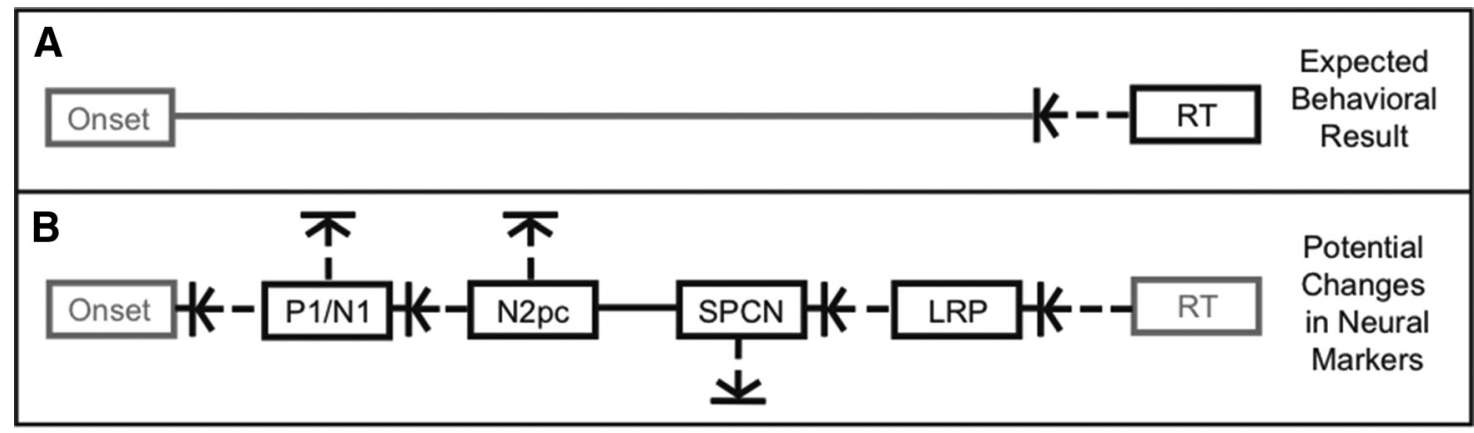

Figure 1. Hypothetical model indicating potential changes in behavior and ERP components in a visual search task after practice. $\boldsymbol{A}$, Response time (RT) is expected to decrease after practice. $\boldsymbol{B}$, Horizontal arrows indicate potential latency shifts in the N1, N2pc, and LRP components. Vertical arrows indicate potential amplitude changes in the P1/N1, N2pc, and SPCN components.

corded on days 1 and 5) to explore plasticity in the neural mechanisms underlying the expected practice-induced reduction in behavioral response time (Fig. 1A).

We assessed changes in four hallmark ERP components that reflect the cascade of cognitive processing stages from stimulus to response (Fig. $1 B$ ): (1) the posterior visual N1, a negative-polarity wave (latency $\sim 150 \mathrm{~ms}$ ) that reflects early sensory-evoked processing (Mangun and Hillyard, 1991); (2) the negative-polarity posterior-contralateral N2pc component (latency $\sim 225 \mathrm{~ms}$ ) associated with the shift of attention to a lateralized stimulus location (Luck and Hillyard, 1994a); (3) the sustained posteriorcontralateral negativity (SPCN) component (latency 300-450 $\mathrm{ms}$ ) or contralateral delay activity (CDA) that has been linked to maintenance and manipulation of information in visual working memory (Jolicoeur et al., 2008; Ikkai et al., 2010) and/or to cognitive processing demands for target discrimination (Emrich et al., 2009; Luria and Vogel, 2011); and (4) the motor-related lateralized readiness potential (LRP) that reflects the initiation of a motor response (Coles, 1988).

By comparing the neural activity associated with these cognitive processes before and after the practice regimen, we aimed to elucidate neural plasticity underlying the expected improvements in visual search efficiency. Specifically, we assessed changes in the amplitude and/or latency of these ERP components to reveal how enhancements in sensory processing, attentional orienting, target discrimination, motor initiation, and/or motor execution contribute to improvement in visual search with practice.

\section{Materials and Methods}

\section{Participants}

Nineteen healthy individuals with normal or corrected-to-normal visual acuity and normal color vision were recruited and provided informed consent. All procedures were approved by the Duke University Medical Center Institutional Review Board. All individuals participated in a fiveday visual search practice protocol for approximately 1 hour per day, over 5 consecutive days, beginning on a Monday and ending on the Friday of the same week. Before the start of the experiment on the first day, participants completed a brief $(\sim 5 \mathrm{~min})$ session to become acquainted with the task. Behavioral performance (accuracy and response time) was recorded on all five days, and scalp-recorded EEG was measured on the first and last days of the protocol. Participants provided informed consent and were compensated at the rate of $\$ 15$ per hour.

Data from two participants were excluded from the analyses because of poor behavioral performance (accuracy percentages more than 2 SDs below the group mean). Data from four additional participants were excluded because of producing suboptimal EEG data (excessive eye or muscle artifacts) on one or both of the EEG sessions. Data from the remaining 13 participants (ages 18-35 years, five female) were included in all analyses.

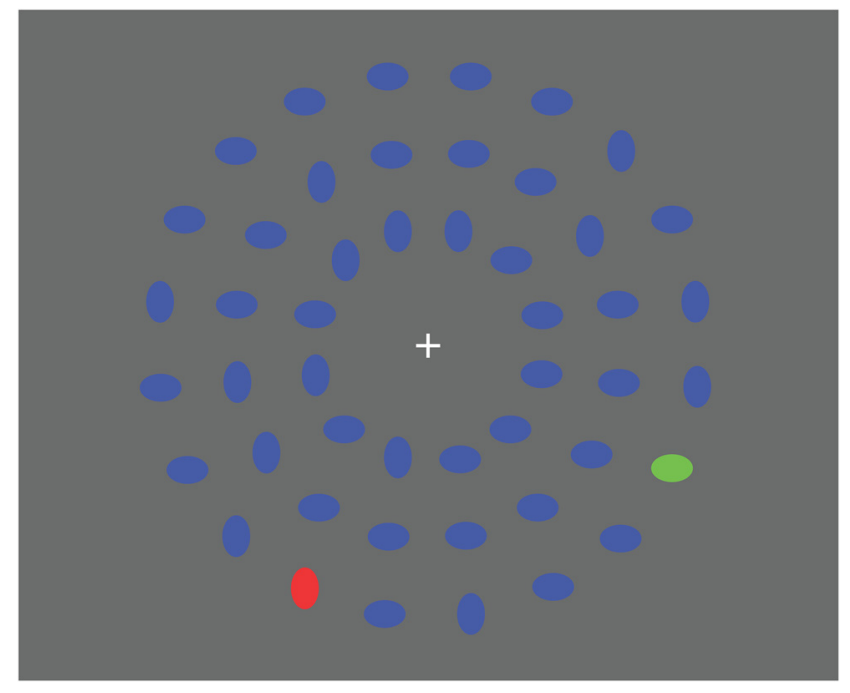

Figure 2. Sample stimulus display. The blue ellipses were distractors, the green ellipse was the relevant color popout target, and the red ellipse was the irrelevant color popout nontarget. Participants responded as to the orientation of the green target ellipse. In this example, a participant would respond by pressing the button corresponding to "horizontal."

\section{Search paradigm}

Stimuli were programmed and presented using the Presentation software suite (Neurobehavioral Systems). During each of the five experimental sessions, participants completed a series of 14 blocks, each consisting of 140 trials and lasting $\sim 4$ min. Thus, each experimental session comprised 1960 trials and lasted $\sim 56 \mathrm{~min}$. Participants were seated without head restraint $\sim 57 \mathrm{~cm}$ from the viewing monitor. A white fixation cross was presented at the center of the screen on a gray background and remained in place for the duration of each experimental block. Each trial consisted of a briefly presented $(50 \mathrm{~ms})$ circular array of 48 colored ellipses of which 46 were blue, 1 was red, and 1 was green (Fig. 2), with each stimulus subtending a visual angle of $1.36 \times 0.91^{\circ}$. Thus, on each trial, there were two color popout stimuli in the array, a green ellipse (the target) and a red ellipse (an irrelevant nontarget popout distractor). These green and red ellipses could appear in one of 10 locations on the lower portion of the array on each trial and always appeared on opposite sides of each other.

Participants were instructed to report the orientation of the green target ellipse as quickly and accurately as possible. Responses were made using the left and right fingers on a game controller to indicate "vertical" or "horizontal" orientations, respectively. Participants were instructed to maintain central fixation during the stimulus presentation to minimize eye movements and preserve visual stimulation consistency. The $50 \mathrm{~ms}$ duration of the presented stimuli was sufficiently brief so as not to allow for a saccade to the target, and individual trials were separated by a stimulus-onset asynchrony that varied between 1300 and $1700 \mathrm{~ms}$. 
A

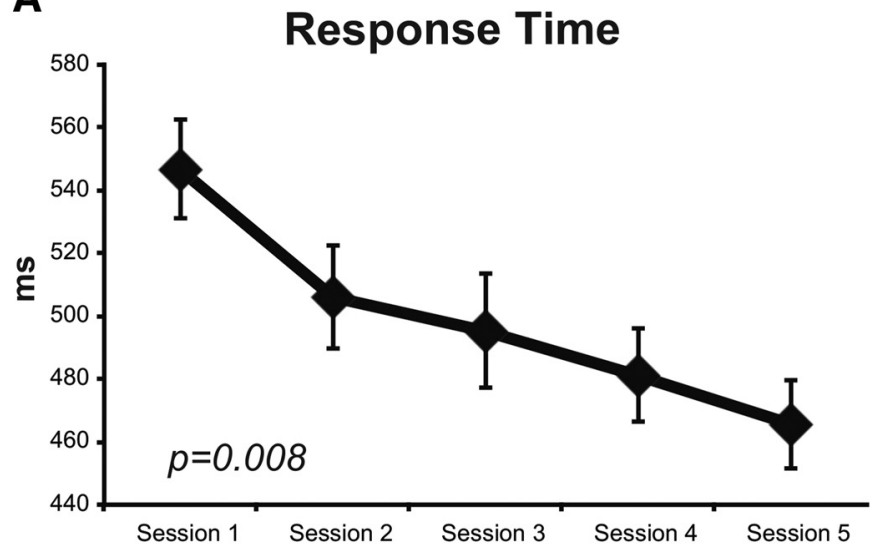

B

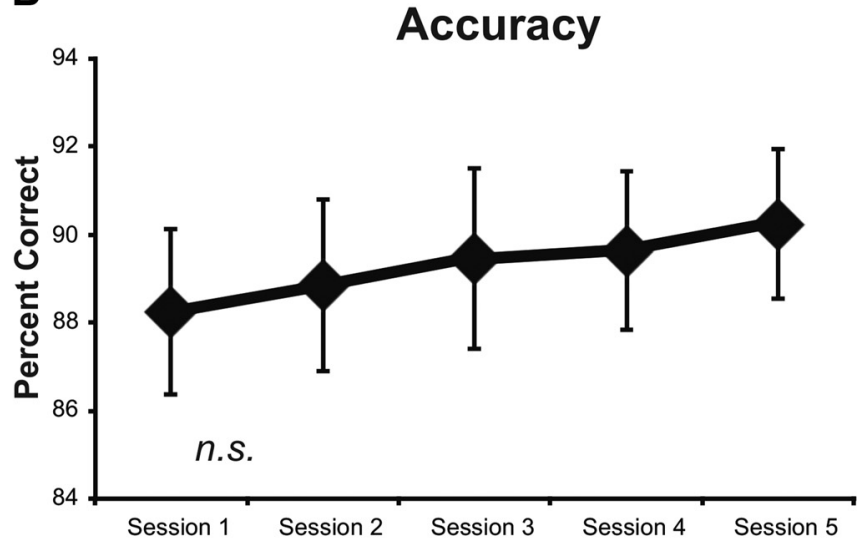

Figure 3. Behavioral results. The response time $(\boldsymbol{A})$ and accuracy values $(\boldsymbol{B})$ are shown across the sessions. The response time decreased significantly over the course of practice, but there was no significant change in accuracy.

The participants' task was to find the green ellipse, shift their spatial attention to this target covertly (i.e., without making an eye movement), and discriminate its orientation (a larger vertical or larger horizontal aspect ratio) with a manual response. The design of the task enabled the extraction of several prototypical ERP markers of the cognitive processes involved in visual search. First, by structuring the search arrays with both a task-relevant target popout (green among blue distractors) and a taskirrelevant nontarget popout (red among blue distractors), we were able to control for early sensory differences in the ERPs (Luck, 2005), while also eliciting robust N2pc and SPCN components. Additionally, the manual responses were executed with the index fingers of the left and right hands (left for vertical targets and right for horizontal targets), thereby allowing assessment of the LRP associated with motor-response initiation. Finally, the final response time was recorded for each trial.

\section{Behavioral data acquisition and analyses}

Behavioral analyses. Behavioral responses were considered accurate if the participant responded with the correct orientation (vertical or horizontal) of the target stimulus between 200 and $1000 \mathrm{~ms}$ after the onset of the array. Response time was recorded as the time between the onset of the stimulus array and the button press for each correctly reported trial. Within-subject differences in accuracy and response time over the course of practice were assessed using repeated-measures ANOVAs. Additional two-tailed, paired $t$ tests were used to compare accuracy and response time between the first (Session 1) and last (Session 5) experimental sessions.

EEG recording and analysis. On Sessions 1 and 5 of the experimental protocol, EEG data were recorded as participants performed the visual search task. Continuous EEG was recorded using a custom extendedcoverage elastic cap with 64 equally spaced channels (Electro-Cap International), which covered the full head from slightly above the eyebrows to below the inion (Woldorff et al., 2002). Impedances of all channels were adjusted to below $5 \mathrm{k} \Omega$; EEG was amplified within a frequency band of $0.016-100 \mathrm{~Hz}$ and digitized at a sampling rate of $500 \mathrm{~Hz}$ per channel (SynAmps; Neuroscan). Eye movements were monitored with vertical and horizontal EOG channels and a closed-circuit zoom-lens camera, and participants were given verbal feedback to encourage fixation on the central cross. Recordings took place in an electrically shielded, soundattenuated, dimly lit experimental chamber.

For each participant, EEG data were selectively averaged to yield ERPs for the various conditions. All channels were re-referenced to the algebraic mean of the two mastoid electrodes. A digital, noncausal, ninepoint (18 ms) running average filter was applied to the ERP averages to reduce signal frequencies $>56 \mathrm{~Hz}$ at our $500 \mathrm{~Hz}$ sampling frequency. Artifact rejection was performed off-line by discarding epochs of the EEG contaminated by eye movements or eye blinks (EOG), excessive musclerelated potentials, drifts, or amplifier blocking. Artifact rejection thresholds were initially set to $\pm 120 \mathrm{uV}$ for vertical eye channels and $\pm 90 \mathrm{uV}$ for all other channels and applied from -200 to $1000 \mathrm{~ms}$ around the presentation of the visual search array. Thresholds were then minimally adjusted for each participant to retain the most trials while eliminating the above sources of contamination, and these artifact-rejection thresholds were then applied via a computer algorithm that was blind to the specific trial types. These parameters led to an average trial-rejection rate of $16.9 \%$, yielding an average of $\sim 1400$ usable trials per participant.

Changes in ERP components were assessed using two-tailed, paired $t$ tests or two-factor ANOVAs of the ERPs and/or ERP difference waves to compare amplitude and/or latency differences between Sessions 1 and 5 . Activity in parietal-occipital channels on the left (PO3, PO5, P3) and right (PO4, PO6, P4) sides of the head was analyzed for changes in the N1 sensory-evoked component. With respect to N2pc-related activity, a parietal-occipital region of interest was also defined, consisting of: one set of three sites on the left side of the head and a set of three corresponding sites on the right (channels PO3, P3, P5, PO4, P4, P6). Activity in these regions was also analyzed for the SPCN/CDA component. Finally, fronto-central channels (FC5, C5, FC6, C6) were used for assessment of the motor-related LRP component.

Visual N1. To examine early sensory processing, EEG activity from parietal-occipital channels was used to calculate the amplitude and latency of the visual N1 component (Mangun and Hillyard, 1991). For this analysis, trials were divided according to whether the target appeared on the left or right side of the screen, and activity in sites contralateral and ipsilateral to the target was assessed. Mean amplitude measures of the N1 for each participant were taken in a 25 ms latency window around the peak of the N1 (140-165 ms) observed in the across-subject grand-averaged ERP, and these values were compared between Sessions 1 and 5 to assess changes in basic sensory processing with practice. Peak latencies of the N1 were also measured for each participant and compared between Sessions 1 and 5 to assess for changes in speed of basic sensory processing.

$N 2 p c$. ERP difference waves reflecting activity associated with the attentional shift-related N2pc component were derived from parietal-occipital scalp sites, calculated as the difference between the activity in posterior electrodes contralateral minus those ipsilateral to the relevant popout target stimulus (Luck and Hillyard, 1994a). The resulting N2pc difference waves were compared between Sessions 1 and 5. The latencies of the peaks of the N2pc components were compared between Sessions 1 and 5 to determine whether the attentional shift to the target occurred sooner, relative to stimulus onset, after practice. These latencies were analyzed using both peak latency measures and onset latency measures. To capture the onset latencies, we calculated the fractional peak latency, the time at which the ERP waveform reached $50 \%$ of its peak amplitude (Luck, 2014). In addition, N2pc amplitudes were compared between Sessions 1 and 5 in the $40 \mathrm{~ms}$ latency window immediately surrounding the average peak latency collapsed over both sessions. Across both sessions, the average peak of the N2pc occurred at $228 \mathrm{~ms}$ (SD, $18 \mathrm{~ms}$ ). Thus, amplitudes were assessed by comparing the mean amplitudes of the N2pc 
activity in the $40 \mathrm{~ms}$ window immediately surrounding the average peak latency.

SPCN. The SPCN (or CDA) component, which occurs after the N2pc, was also computed as a contralateral-minus-ipsilateral difference wave using the same calculation used for the N2pc, but examined in a later time window. The amplitudes of the SPCN component were analyzed in a broad time window after the N2pc component (340-480 ms after the stimulus array) and compared between Sessions 1 and 5 to assess changes in target-discrimination processing. Previous research has indicated that this component tends to become smaller for target-discrimination processes during search that are easier relative to those that are more taxing (Emrich et al., 2009; Luria and Vogel 2011).

LRP. Activity associated with the motorrelated LRP component was calculated timelocked to both the stimulus array and the behavioral response. For both cases, this activity was calculated as the voltage difference between electrodes over the motor cortices contralateral versus ipsilateral to the hand used to execute the response on each trial (e.g., left for vertical orientation, right for horizontal orientation). For the LRP responses relative to the search array presentation, stimulus-locked activity over the motor cortex ipsilateral to the response hand was subtracted from the activity at the corresponding scalp site contralateral to the response hand, and the resulting LRP difference waves were compared between Sessions 1 and 5 to assess whether participants began to prepare motor responses more quickly after practice. These latencies were analyzed using both peak latency measures and onset latency measures (the latter using a 50\% fractional peak latency calculation). The amplitudes of the LRP components between the two sessions were also compared, assessed in a $40 \mathrm{~ms}$ window around the grand-averaged peak of the component observed in each session. The response-locked LRP was derived at the same sites and using the same contralateral-minusipsilateral computation as the stimulus-locked LRP but was extracted such that it was time-locked to the behavioral response rather than to the stimulus. Baseline correction of the response-locked LRPs was performed by subtracting the mean amplitude from 1.2 to $1 \mathrm{~s}$ before the motor response. Because trials with responses longer than $1000 \mathrm{~ms}$ were excluded, this approach ensured that there was no overlap between the baseline correction period and the stimulus-evoked ERPs. As with the stimulus-locked LRP, we used the fractional peak latency for statistical analyses of the response-locked activity.

\section{Results}

\section{Behavioral results: response time and accuracy}

As expected, participants responded more quickly after practice. There was a significant main effect of session on response time $\left(F_{(4,12)}=3.76, p=0.008\right)$ with an average decrease of $81.1 \mathrm{~ms}$ and a significant difference between Session 1 [546.7 ms (SD, 56.2)] and Session 5 [465.7 $\mathrm{ms}(\mathrm{SD}, 50.8) ; t_{(12)}=10.01, p<0.001$; Fig. $3 A]$. Improvement was observed in all participants, with the response time decreases ranging from 30.6 to $128.6 \mathrm{~ms}$ across the sample. Additionally, the average SD of individuals' response times was $102.4 \mathrm{~ms}$ in Session 1 and $80.0 \mathrm{~ms}$ in Session $5\left(t_{(12)}=\right.$ $8.70, p<0.001)$.

There were no differences in accuracy across the five sessions $\left(F_{(4,12)}=0.16, p=0.96\right)$ and no difference in accuracy in the
Table 1. Means and SDs of peak amplitudes and latencies of the peak of the N1 component

\begin{tabular}{llll}
\hline & Session 1 & Session 5 & Statistics \\
\hline Amplitude $^{a}$ & & & \\
$\quad \begin{array}{lll}\text { Contralateral to target } \\
\text { Ipsilateral to target }\end{array}$ & $-4.63 \mu \mathrm{V}(2.16)$ & $-5.96 \mu \mathrm{V}(2.90)$ & $t_{(12)}=2.45, p=0.03$ \\
$\begin{array}{c}\text { Latency } \\
\text { Contralateral to target }\end{array}$ & $154.7 \mathrm{~ms}(13.7)$ & $154.8 \mathrm{~ms}(12.2)$ & $t_{(12)}=0.07, p=0.95$ \\
Ipsilateral to target & $152.2 \mathrm{~ms}(13.7)$ & $153.7 \mathrm{~ms}(12.0)$ & $t_{(12)}=0.94, p=0.36$ \\
\hline
\end{tabular}

${ }^{a}$ Means and SDs of peak amplitudes of the sensory-evoked $\mathrm{N} 1$ component, collapsed across left and right targets. ${ }^{b}$ Means and SDs of latencies of the peak of the $\mathrm{N} 1$ component in response to targets on the left and right sides of the display.

direct comparison between Session 1 [88.2\% (SD, 6.8\%)] and Session 5 [90.2\% (SD, 6.1\%); $t_{(12)}=1.01, p=0.33$; Fig. $\left.3 B\right]$; thus, participants maintained the same level of accuracy for the duration of practice. The fact that response time decreased significantly with no sacrifice in accuracy suggests that the improvement in response speed was not the result of a speed/accuracy tradeoff.

\section{Electrophysiological markers}

Early visual sensory processing: N1 effects

Mean amplitudes around the N1 peak (latency window, 140-165 $\mathrm{ms}$ ) were significantly larger in Session 5 than in Session 1 for sites both contralateral $\left(t_{(12)}=2.45, p=0.03\right)$ and ipsilateral $\left(t_{(12)}=\right.$ 2.30, $p=0.04$ ) to the target (Fig. $4 A$; Table 1 , amplitude). This overall amplitude increase in the N1 component bilaterally sug- 
A
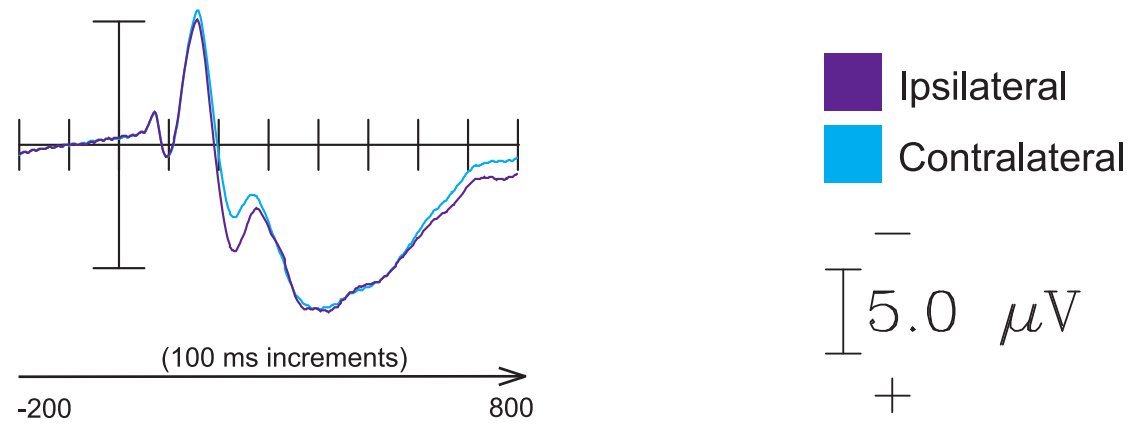

B

\section{Contralateral minus Ipsilateral}
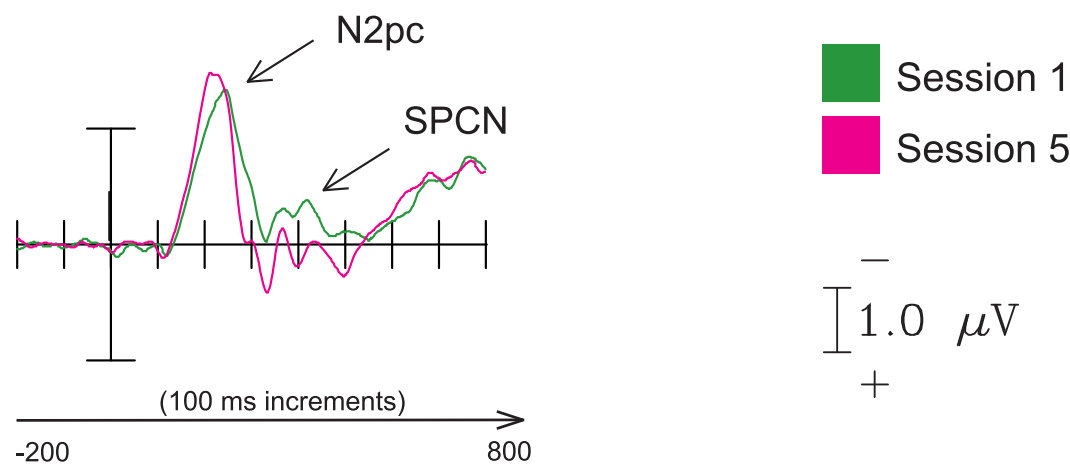

C

Session 1
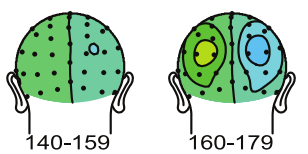

Distribution of N2pc-related activity

Session 5
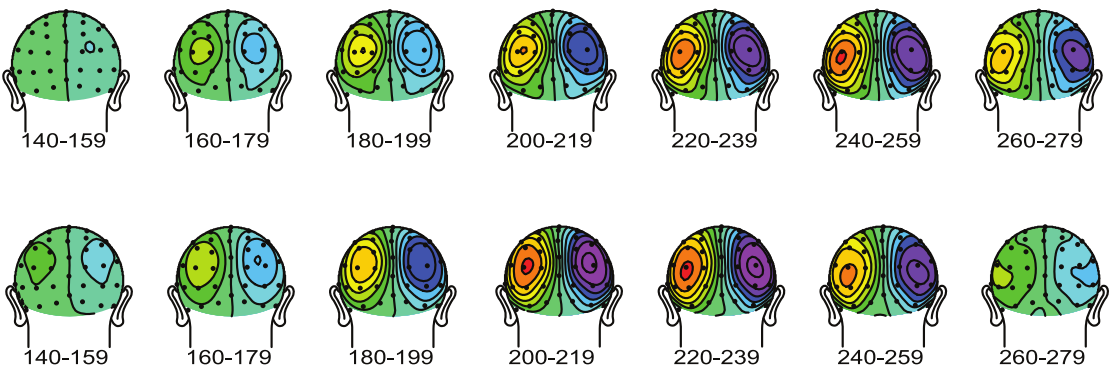

$2 \mu \mathrm{V}$

Time (in ms)
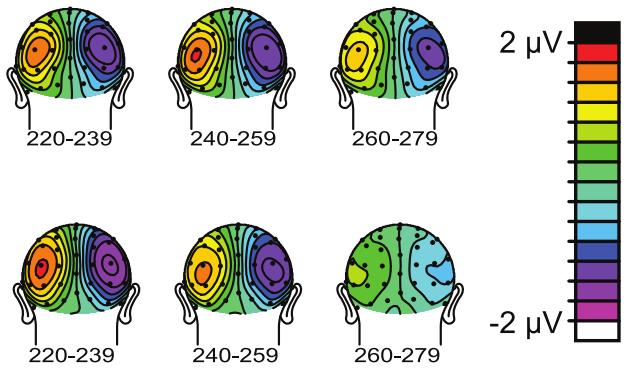

D

Distribution of SPCN-related activity

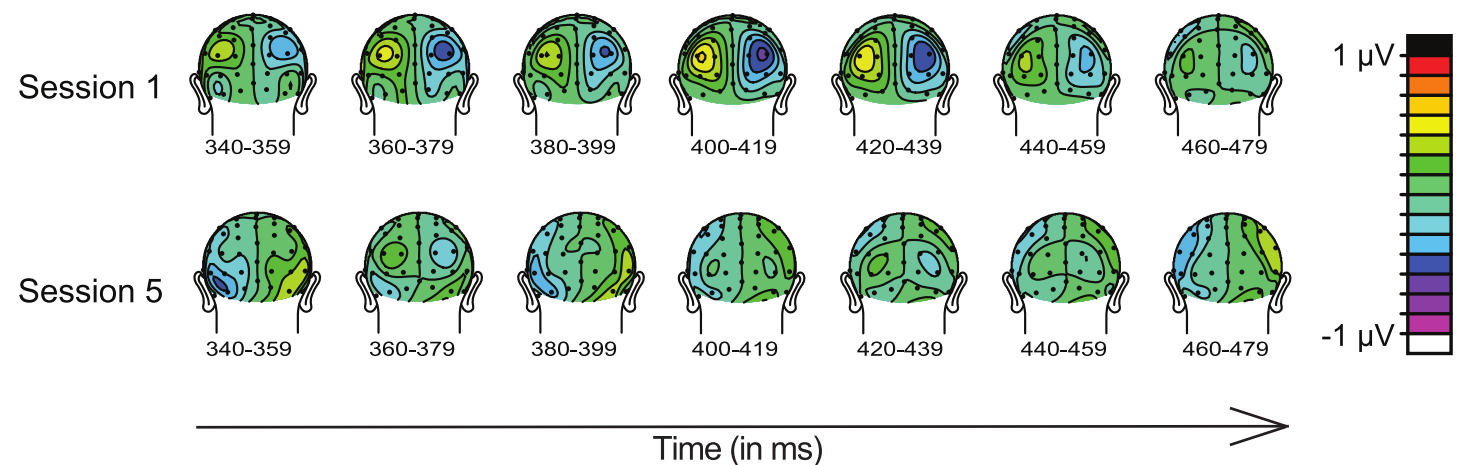

Figure 5. Effects on the N2pc and SPCN lateralized components, reflecting attentional orienting and target discrimination difficulty, respectively. $A$, ERP traces of activity used to calculate the difference waves for deriving the N2pc and SPCN components (contralateral vs ipsilateral to the target popout). $\boldsymbol{B}$, Difference waves displaying N2pc and SPCN components in the posterior parietal-occipital regions of interest. C, Distribution of N2pc-related activity over the scalp for Sessions 1 and 5. D, Distribution of SPCN-related activity over the scalp for Sessions 1 and 5. 
Table 2. Means and SDs of the amplitudes and latencies of the N2pc component

\begin{tabular}{lccl}
\hline & Session 1 & Session 5 & Statistics \\
\hline Amplitude $^{a}$ & $-1.23 \mu \mathrm{V} \mathrm{(0.42)}$ & $-1.44 \mu \mathrm{V}(0.45)$ & $t_{(12)}=2.42, p=0.032$ \\
Peak latency $^{b}$ & $236 \mathrm{~ms}(18)$ & $217 \mathrm{~ms}(13)$ & $t_{(12)}=3.88, p=0.002$ \\
Onset latency & $190 \mathrm{~ms}(18)$ & $181 \mathrm{~ms}(15)$ & $t_{(12)}=3.36, p=0.006$
\end{tabular}

${ }^{a}$ Means and SDs of the amplitudes of the N2pc component, collapsed across left and right targets (contralateral vs ipsilateral to the target popout).

${ }^{b}$ Means and SDs of the latencies of the N2pc component, collapsed across left and right targets (contralateral vs ipsilateral to the target popout).

gests a generalized enhancement of neural responses to the stimulus arrays with practice [also shown by the main effect of session in the corresponding $\left.\operatorname{ANOVA}\left(F_{(1,12)}=5.66, p=0.035\right)\right]$.

The analyses also showed that the N1 peak amplitudes (latency window, 140-165 ms) were significantly larger in sites contralateral to the target stimulus than in sites ipsilateral to the target stimulus in both sessions $\left(t_{(12)}=4.18, p<0.002\right)$. Given that the stimulus arrays were controlled for left-right physical stimulus differences (a color popout on each side), these effects were likely related to the analysis including the early part of the contralaterality of the N2pc, which began in the middle of the N1 latency range (see below). There was also a trend for an interaction between laterality and session, but this did not reach significance; thus, there was not a significant effect of practice on the N1 laterality. No differences in the peak latency of the $\mathrm{N} 1$ component were observed between any of the conditions (Table 1 , latency).

Allocation of attention: N2pc peak latency and amplitude

After practice, the N2pc component peaked significantly sooner (by $\sim 19 \mathrm{~ms}, t_{(12)}=3.88, p=0.002$; Fig. $5 A-C$; Table 2, peak latency). Analysis of the onset latency measure [fractional peak latency] also showed a significantly earlier onset with practice (by $\left.9 \mathrm{~ms}, t_{(12)}=3.36, p=0.005\right)$. There was also a significant increase in the amplitude of the peak of the N2pc component in the latency window centered around the average time of the peak across subjects $\left(t_{(12)}=2.42, p=0.032\right)$. Furthermore, there was a relationship between individual participants' initial N2pc peak latencies and the amount of N2pc peak latency change with practice $(r=0.72, p=0.006)$. Participants with initially later N2pc latencies showed greater improvement after practice, suggesting that there may have been more room to improve in those cases.

\section{Target discrimination: SPCN amplitude}

There was a significant decrease in the mean amplitude of the SPCN component after practice [Session $1,-0.29 \mu \mathrm{V}(\mathrm{SD}, 0.9)$; Session 5, $0.03 \mu \mathrm{V}(\mathrm{SD}, 0.9)$; $t_{(12)}=4.05, p=0.002$; Fig. $\left.5 A, B, D\right]$, suggesting that practice facilitated target-discrimination processes requiring retention and/or manipulation of information in visual short-term memory.

Motor-response preparation and execution: LRP onset latencies The onset of the stimulus-locked LRP component was significantly earlier [fractional peak latency difference $\sim 41 \mathrm{~ms}(\mathrm{SD}, 20$ $\mathrm{ms})$ ] in Session 5 than in Session $1\left(t_{(12)}=7.4, p<0.0001\right.$; Fig. 6 , Table 3), reflecting earlier initiation of the motor response after practice. This was further supported by the latency of the peak amplitudes of the LRP component also being significantly earlier [by $\sim 47 \mathrm{~ms}$ (SD, $30 \mathrm{~ms})$ ] in Session 5 than in Session $1\left(t_{(12)}=\right.$ $5.7, p=0.0001)$. No difference was observed between the amplitude of the LRP component in Sessions 1 and 5. The onset of the response-locked LRP was closer in time to the behavioral response [fractional peak latency difference $\sim 21 \mathrm{~ms}(\mathrm{SD}, 19$ $\mathrm{ms})$ ] in Session 5 compared with Session $1\left(t_{(12)}=4.14, p=\right.$ 0.0014; Fig. 6), suggesting that practice led to more rapid mo- tor execution after motor-response initiation. The amplitude of the LRP did not significantly change with practice.

\section{Summary of results}

The results from this study delineate learning and plasticity in key phases of the neurocognitive processing chain associated with behavioral improvements in visual search performance with practice. Over the course of five sessions of practice on a visual search popout task, participants became significantly faster to detect and discriminate targets, without any detriment in accuracy. The electrophysiological measures of brain activity showed that this behavioral performance improvement was accompanied by a significant bilateral increase in the overall amplitude of the sensory-evoked visual N1 ERP component to the array, an increase in amplitude and a shortening in latency of the attention-shifting-sensitive N2pc, a significant decrease in the amplitude of the SPCN reflecting reduced target-discrimination resources demands, a significant quickening of the onset latency of the LRP reflecting faster motor-response initiation, and a significant quickening of the time between the motorresponse initiation and response time.

\section{Discussion}

We aimed to elucidate how various stages of neurocognitive processing involved in visual search are improved with practice on a feature-popout search task. After practice on the task, we observed a robust improvement in behavioral response times and changes in several ERP indices that reveal the underlying neuroplasticity associated with learning in this task. In the following sections, we discuss the logic behind this experimental design and how the observed practice effects demonstrate learning in the distinct cognitive processing stages underlying visual search.

\section{Using ERPs to infer learning in visual search mechanisms}

ERPs provide a high-temporal-resolution measure of brain activity that has been widely used to study the cognitive mechanisms underlying visual search abilities (Luck and Hillyard, 1994b; Jolicoeur et al., 2008 Luck and Hillyard, 1994a; Hickey et al., 2009). In the current study, we used a feature-popout search in which the target elements possessed a feature that was absent from all distractors, thus capturing attention and providing high-fidelity ERP components with minimal trial-to-trial timing variability. The present design also required hand-specific manual responses and therefore enabled extraction of the LRP activity reflecting motor response-related neural responses, derived with both stimulus-locked and response-locked analyses, thereby providing measures spanning the full cascade of processes from stimulus processing to response execution. This approach differs from previous recent studies that have investigated practice-related changes in visual search using conjunction search stimuli (Hamame et al., 2011; An et al., 2012). Whereas those studies have revealed some context-specific enhancements in the N2pc and P3 components, the use of conjunction searches that require numerous fixations make it difficult to infer the precise timing of brain responses and only offer a limited view of the full cascade of mechanisms that may change as a result of practice.

\section{Sensory processing}

After practice, we did not observe a change in the latency of the $\mathrm{N} 1$ sensory component, indicating that speeding of basic visual sensory processing did not contribute to the ultimate speeding of response time. We did, however, observe a significant bilateral increase in the amplitude of the N1 component after practice, suggesting enhancement of overall early sensory responses to the entire array with practice. A prior study did not find a robust 
A

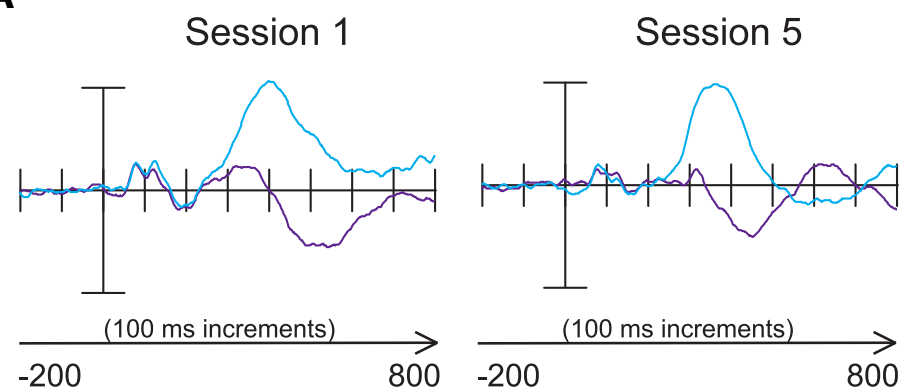

Ipsilateral

Contralateral

]$_{+}^{2.0 \mu \mathrm{V}}$
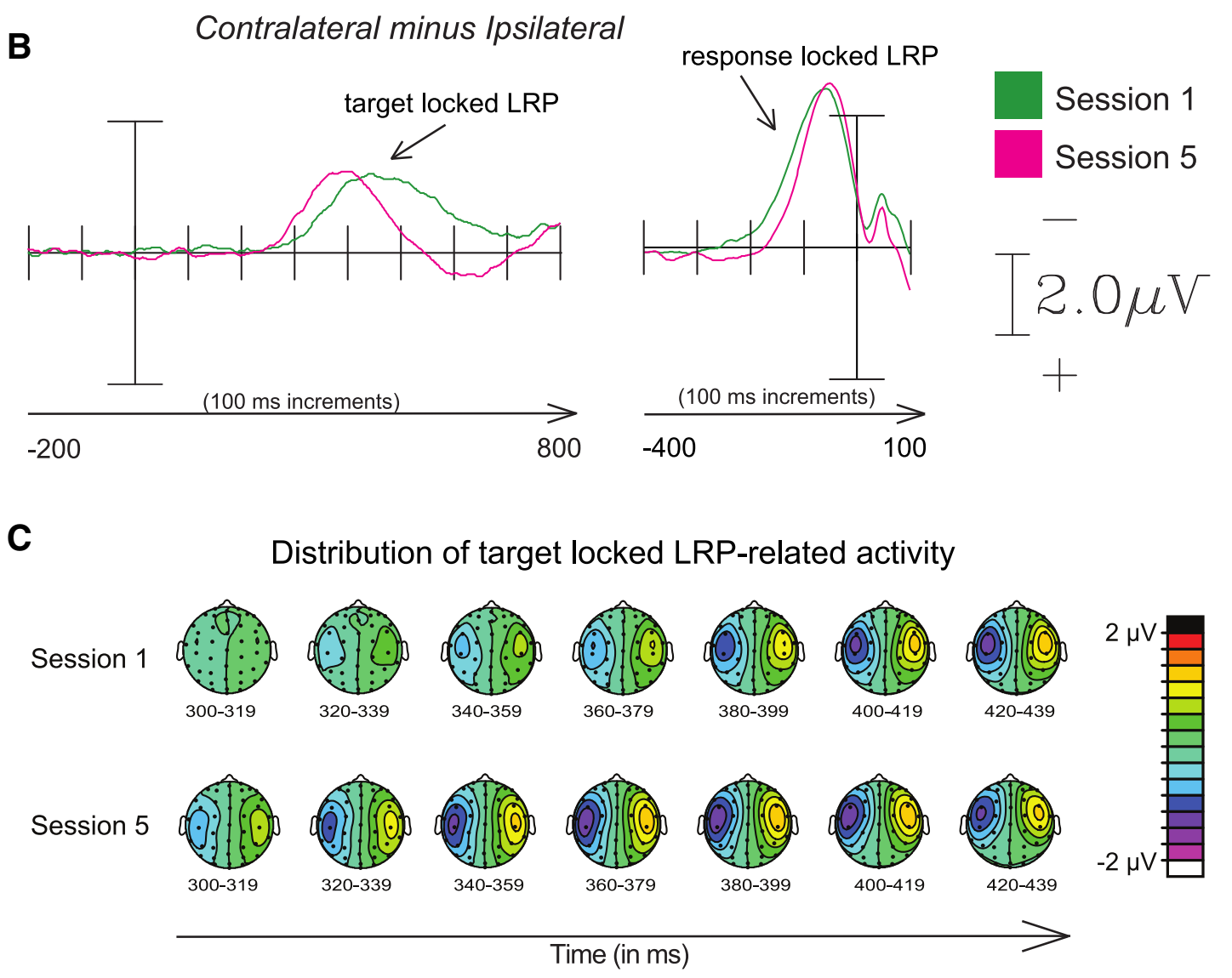

Figure 6. Effects on the LRP component, reflecting stimulus-locked motor preparation activity and response-locked motor preparation activity. A, ERP traces of activity used to calculate contralateral versus ipsilateral difference waves used for deriving the LRP component (contralateral vs ipsilateral to the hand used for the motor response), time-locked to the onset of the stimulus array (stimulus-locked, left traces) and to the response time (response-locked, right traces). $\boldsymbol{B}$, Difference waves displaying the LRP component. $\boldsymbol{C}$, Distribution of LRP-related activity over the scalp for Sessions 1 and 5.

Table 3. Means and SDs of the onset and peak latencies of the LRP component

\begin{tabular}{cccl}
\hline & Session 1 & Session 5 & Statistics \\
\hline Stimulus-Locked & & & \\
Onset latency & $362 \mathrm{~ms}(29)$ & $321 \mathrm{~ms}(27)$ & $t_{(12)}=8.68, p<0.0001$ \\
Peak latency & $443 \mathrm{~ms}(38)$ & $395 \mathrm{~ms}(40)$ & $t_{(12)}=5.7, p=0.0001$ \\
Response-Locked & & & \\
Onset latency & $-131 \mathrm{~ms}(20)$ & $-109 \mathrm{~ms}(18)$ & $t_{(12)}=4.14, p=0.001$ \\
Peak latency & $-60 \mathrm{~ms}(14)$ & $-53 \mathrm{~ms}(19)$ & $t_{(12)}=1.1, p=$ n.s. \\
\hline
\end{tabular}

change in N1 amplitude in response to practice in a conjunction search task (Hamame et al., 2011) and suggested that cortical reorganization at this early sensory processing stage may not have been necessary or beneficial for such a serial search task, whereas such early-processing plasticity did seem to be at play for our feature-singleton target-popout task. The larger N1s in Session 5 in the present study could be the result of directing more attention toward the incoming stimulus array after practice, as stronger attention to stimulus input has been shown to produce larger N1s (Luck et al., 1994a). We also observed a small degree of contralaterality of the early sensory-evoked N1 component that distinguished targets from nontargets (slightly larger contralateral to the target), but we did not find a significant training effect on this contralaterality.

\section{Allocation of attention}

The N2pc is a parietal-occipital electrophysiological component (latency, 175-300 ms) that reflects a lateralized shifting and focusing of attention to a specified target item (Luck and Hillyard, 
1994a; Hopf et al., 2000). After practice on the current paradigm, we observed larger amplitudes of the N2pc, indicating enhanced attentional orienting. This result is consistent with the results of two recent practice studies with conjunction visual search (Hamame et al., 2011; An et al., 2012), which also reported a larger N2pc after practice. Additionally, we observed a significant shortening in the latency of the onset and of the peak of the N2pc component after practice, which was not observed in the prior studies. It is possible that this latency effect was observed here, but not in the prior studies using conjunction searches, because the tighter timing and minimal trial-to-trial variability of the responses in our popout search task enabled a more precise temporal measure of the attentional shifting process. Alternatively, this latency effect may reflect differential practice effects for popout searches versus conjunction searches. In either case, this latency effect suggests that it is possible for the rapid process of attentional orienting to a feature-popout stimulus to become even faster with practice.

Importantly, response time improved by $\sim 81 \mathrm{~ms}$ after practice, and the N2pc shifted by only 9-19 ms (depending on the measure). Thus, it should be noted that the majority of processing speed improvement appears to occur later in the cognitive cascade, although a larger N1 and N2pc earlier in the processing stream could certainly contribute to the acceleration of later processing. Finally, because there was no latency shift in the early sensory-evoked N1 component with practice, the N2pc was the earliest point in the cascade of search mechanisms in which learning appeared to speed the neural processing. The robust bilateral enhancement of the amplitude of the N1 suggests that practice led to enhanced sensory processing to the entire stimulus array, perhaps because of enhanced overall attentional focus, which may have then facilitated and accelerated the next stage of processing, namely the detection of and orienting toward the popout target.

\section{Target discrimination}

The SPCN, or sustained posterior-contralateral negativity (also often termed the CDA, or contralateral delay activity), is a lateralized ERP component associated with cognitive processing that occurs after attentional allocation to a lateralized target stimulus (Jolicoeur et al., 2008). The amplitude of the SPCN has been related to the cognitive resources required to complete the discrimination task; specifically, amplitude increases are observed in visual search contexts that place greater demands on working memory and/or target discrimination processes (Emrich et al., 2009; Eimer and Kiss, 2010; Luria and Vogel 2011). Unlike the $\mathrm{N} 2 \mathrm{pc}$, the SPCN does not typically show a peak but rather displays sustained activity occurring for several hundred milliseconds while visual information is being processed.

We observed a substantial decrease in the amplitude of the SPCN after practice. Participants searched the same displays and completed the same task over the course of the practice protocol, so the absolute level of difficulty of the task itself remained constant. Accordingly, the SPCN amplitude decrease likely reflects learning for the vertical-horizontal discrimination of the popout target such that it became less demanding after practice, thus requiring fewer neural resources to perform this discrimination.

\section{Motor-response preparation and execution}

The LRP, or lateralized readiness potential, is a well characterized, centrally distributed, negative wave measured over the motor cortices (Coles, 1988). This component reflects preparation for a motor response, thus providing a high-temporal-resolution marker of the initiation of voluntary movement. We observed a significant shortening in the onset latency of the stimulus-locked LRP after practice (by $\sim 41 \mathrm{~ms}$ ). The latency shift of stimulus-locked LRP shows that training shortened the amount of time between the onset of the stimulus and the onset of response preparation. The response-locked LRP additionally demonstrated an improvement in response-execution efficiency, reducing the time between motorresponse initiation and actual motor output by $\sim 20-30 \mathrm{~ms}$.

\section{Temporal changes in cognitive stages}

The earliest temporal change after practice was observed in the $\mathrm{N} 2$ pc (earlier in its onset by $9 \mathrm{~ms}$, in its peak by $19 \mathrm{~ms}$ ), reflecting a faster attentional shift to the target. An additional change in latency was apparent in the onset of the LRP, which occurred $\sim 41$ $\mathrm{ms}$ faster relative to stimulus onset after practice. Thus, earlier preparation for the motor response after attentional orienting contributed approximately $\sim 20-30 \mathrm{~ms}$ to the overall improvement in response time, facilitation that likely suggests a more efficient target discrimination process after practice, as reflected by the reduction in the SPCN. Accordingly, the latency shifts observed in the N2pc and the LRP together account for $\sim 41$ of the $\sim 81 \mathrm{~ms}$ change in response time, suggesting that additional speeding of processing occurred between the initiation and execution of the motor response. Finally, increased efficiency in response execution (as reflected by the reduction in time between the onset of the LRP in the response-locked LRP and the actual motor output, measured by $50 \%$ fractional peak latency) specifically accounted for an additional 20-30 ms of the total observed behavioral improvement.

\section{Conclusions}

Our primary goal was to determine which cognitive processes underlying visual search are enhanced with practice and to delineate their relative contributions to improved behavioral performance. In parallel with a marked decrease in behavioral response time with practice, we observed a number of changes in the underlying neural activity associated with specific cognitive mechanisms leading up to the behavioral response, namely, enhanced early sensory processing to the visual search array, enhanced and earlier attention orienting to the popout target item, decreased need of resources required for target discrimination, more rapid initiation of motor-response preparation, and more rapid execution of the motor response after that initiation.

Visual search is a complex but critical cognitive function that requires a cascade of component processes to be performed successfully and effectively. The present findings elucidate specific practice-induced changes in the component neurocognitive stages underlying visual search and offer a principled method for probing the neural mechanisms underlying learning in this essential cognitive ability.

\section{References}

An A, Sun M, Wang Y, Wang F, Ding Y, Song Y (2012) The N2pc is increased by perceptual learning but is unnecessary for the transfer of learning. PLoS One 7:e34826. CrossRef Medline

Clark K, Cain MS, Adamo SH, Mitroff SR (2012) Overcoming hurdles in translating visual search research between the lab and the field: the influence of attention, learning, and motivation on visual search. Nebr Symp Motiv 59:147-181. Medline

Coles MGH (1988) Modern mind-brain reading: psychophysiology, physiology, and cognition. Psychophysiology 26:251-269. Medline

Corbetta M, Shulman GL (2002) Control of goal-directed and stimulus- 
driven attention in the brain. Nat Rev Neurosci 3:201-215. CrossRef Medline

Duncan J, Humphreys GW (1989) Visual search and stimulus similarity. Psychol Rev 96:433-458. CrossRef Medline

Eckstein MP (2011) Visual search: a retrospective. J Vis 11:pii:14:1-36. CrossRef Medline

Eimer M, Kiss M (2010) The top-down control of visual selection and how it is linked to the N2pc component. Acta Psychol 135:100-102. CrossRef Medline

Emrich SM, Naseem A, Pratt J, Ferber S (2009) Visual search elicits the electrophysiological marker of visual working memory. PLoS One 4:e8042. CrossRef Medline

Hamame CM, Cosmelli D, Henriquez R, Aboitiz F (2011) Neural mechanisms of human perceptual learning: electrophysiological evidence for a two-stage process. PLoS One 6:e19221. CrossRef Medline

Hickey C, Di Lollo V, McDonald JJ (2009) Electrophysiological indices of target and distractor processing in visual search. J Cogn Neurosci 21:760775. CrossRef Medline

Hopf JM, Luck SJ, Girelli M, Hagner T, Mangun GR, Scheich H, Heinze HJ (2000) Neural sources of focused attention in visual search. Cereb Cortex 10:1233-1241. CrossRef Medline

Ikkai A, McCollough AW, Vogel EK (2010) Contralateral delay activity provides a neural measure of the number of representations in visual working memory. J Neurophysiol 103:1963-1968. CrossRef Medline

Jolicoeur P, Brisson B, Robitaille N (2008) Dissociation of the N2pc and sustained posterior contralateral negativity in a choice response task. Brain Res 1215:160-172. CrossRef Medline
Luck SJ (2005) An introduction to the event-related potential technique. Cambridge, MA: MIT.

Luck SJ (2014) An introduction to the event-related potential technique, Ed 2. Cambridge, MA: MIT

Luck SJ, Hillyard SA (1994a) Electrophysiological correlates of feature analysis during visual search. Psychophysiology 31:291-308. CrossRef Medline

Luck SJ, Hillyard SA (1994b) Spatial filtering during visual search: evidence from human electrophysiology. J Exp Psychol Hum Percept Perform 20: 1000-1014. CrossRef Medline

Luria R, Vogel EK (2011) Visual search demands dictate reliance on working memory storage. J Neurosci 31:6199-6207. CrossRef Medline

Mangun GR, Hillyard SA (1991) Modulations of sensory-evoked brain potentials indicate changes in perceptual processing during visual-spatial priming. J Exp Psychol 17:1057-1074. Medline

Nakayama K, Martini P (2011) Situating visual search. Vision Res 51:15261537. CrossRef Medline

Sigman M, Gilbert CD (2000) Learning to find a shape. Nat Neurosci 3:264-269. CrossRef Medline

Sireteanu R, Rettenbach R (1995) Perceptual learning in visual search: fast, enduring, but non-specific. Vision Res 35:2037-2043. CrossRef Medline

Treisman AM, Gelade G (1980) A feature integration theory of attention. Cogn Psychol 12:97-136. CrossRef Medline

Wetter OE (2013) Imaging in airport security: past, present, future, and the link to forensic and clinical radiology. J Forensic Radiol Imaging 1:152160. CrossRef

Woldorff MG, Liotti M, Seabolt M, Busse L, Lancaster JL, Fox PT (2002) The temporal dynamics of the effects in occipital cortex of visual-spatial selective attention. Brain Res Cogn Brain Res 15:1-15. CrossRef Medline 